\title{
L'ECHEC DE L'ACTION POLICIERE ANTI-KULUNA ET LA TENTATIVE D'UNE NOUVELLE APPROCHE ERADICATRICE EN REPUBLIQUE DEMOCRATIQUE DU CONGO
}

\author{
Par MILOLO NSENDA Martin*
}

\begin{abstract}
RESUME
La police nationale congolaise (PNC) a reçus la mission de sécurisation des personnes et de leurs biens en plus du maintien et/ou rétablissement de l'ordre public en République Démocratique du Congo à l'instar des forces armées de la République qui s'occupe de la défense et de l'intégrité du territoire nationale contre les agressions externes et les rebellions internes. Cependant la PNC fait face aux situations inédites et complexes dans la réalisation de sa mission qui lui impose une adaptation ; il s'agit notamment du banditisme urbains mieux connus sous l'appellation "phénomène kuluna ». Les Kulunas sont les criminels majoritairement jeunes qui se trouvent dans les villes congolaises et opèrent le jour comme la nuit en extorquant l'argent et autres bien de valeurs de la populaire. Pour faire à cette insécurité urbaine, le Gouvernement a décrété plusieurs opérations exécutées par la police nationale, notamment "Likofi » et "Ingeta " pour éradiquer le phénomène Kuluna en RDC. Toutes les opérations et initiatives se sont soldées par un échec du fait notamment du manque des matériels de travails nécessaires et d'un mauvais traitement des policiers constituant ainsi la cause de la démobilisation policière. Face à cet échec, la présente étude propose une autre stratégie qui consiste à résoudre les causes de la délinquance des Kulunas notamment la formation aux métiers et l'enrôlement dans les ateliers afin de briser leur précarité financière qui est la première cause ce phénomène.
\end{abstract}

\begin{abstract}
The Congolese national police (CNP) received the mission of securing people and their things in addition to the maintenance and / or restoration of public order in the Democratic Republic of Congo like the armed forces of the Republic which takes care of the defense and the integrity of the national territory against external aggressions and internal rebellions. However, the CNP faces new and complex situations in carrying out its mission which requires it to adapt; these include urban banditry better known as the "kuluna phenomenon ». Kulunas are the predominantly young criminals who are found in Congolese cities and operate day and night by extorting money and other valuables from the people.
\end{abstract}

* Assistant et chercheur à la Faculté de Droit de 1'Université de Kinshasa. E-mail : milolonsenda@gm ail.com, Téléphone : +243813263827. 
To end with this urban insecurity, the Government has decreed several operations carried out by the national police, like "Likofi " and "Ingeta " to eradicate the Kuluna phenomenon in the DRC. All those operations and initiatives ended in failure, notably due to the lack of necessary working materials and bad treatment of the police, thus constituting the cause of police demobilization. Faced with this failure, the present study proposes another strategy which consists in resolving the causes of delinquency of the Kulunas in particular training in the trades and enrollment in the workshops in order to break their financial precariousness which is the first cause of this phenomenon.

\section{INTRODUCTION}

Depuis les années 2006 et 2007, un terrorisme inédit monte en puissance quotidiennement dans les grandes villes congolaise (RDC) avec une puissance particulière à Kinshasa, mieux connus sous le nom du phénomène Kuluna. Les Kulunas sont des jeunes délinquants récidivistes qui trouvent leur survie dans la souffrance de la population à travers les vols, viols et extorsions accompagnés des armes blanches et reconnaissables par leurs coiffures extraordinaires, les tatouages et le grossissement non sportif des muscles. La police nationale congolaise a initié plusieurs opérations anti-kuluna depuis 2010 pour éradiquer ce phénomène aux registres desquelles, l'on retrouve Likofi 1 et 2 en 2013 à Kinshasa. Mais en depuis de celles-ci, le phénomène ne cesse de monter en puissance et la population vis dans une insécurité notoire suite à cette réalité déplorable.

Ce sombre tableau prouve les limites ou l'échec de l'action de la police dans la lutte contre le phénomène kuluna. L'on peut dès lors se demandé comment et pourquoi la police national qui a reçu la mission de sécuriser la population et leurs biens se révèle-elle impuissante face aux kuluna qui continuent à semer la terreur dans la population ? Dans une approche multi disciplinaire, la présente réflexion vise à démontrer d'une part que l'échec de la police dans la lutte anti-kuluna résulte de plusieurs défis techniques, logistique et stratégiques auxquelles elle est confrontée dans l'exécution de la sa mission et d'autre part à proposer un diagnostic approfondie des causes à l'origine du phénomène et proposer au gouvernement d'envisager une nouvelle approche éradicatrice du phénomène notamment les recyclages des kuluna aux métiers professionnels afin de leurs permettre de gagner leurs vies par le travail, car la majorité de ces jeunes se lancent dans la criminalité pour des raisons économiques qui résultent de l'apartheid économique qui se vis dans notre pays.

\section{A. Emergence du phénomène kuluna en République Démocratique du Congo}

La République Démocratique du Congo (RDC) connait ces dix dernières années, un taux d'insécurité le plus élevé de l'Afrique central ${ }^{1}$. A côté des éternels groupes armées qui me-

1 Rapport circonstanciel sur l'insécurité en RDC, AZADHO, Kinshasa 2011, p.5. 
nacent la quiétude sur une grande partie du territoire national notamment à l'Est ${ }^{2}$ et au centre du pays, une criminalité aux allures terroriste bat le record dans les grandes villes congolaises avec une cruauté particulière à Kinshasa la capitale, désignée sous le nom du phénomène Kuluna ${ }^{3}$. Un casse-tête pour l'Etat qui aura tout tenté sur le plan répressif sans effet, car les kulunas ne cessent de semer la désolation. D'aucuns pense que ce phénomène qui s'attaquent aux paisibles citoyens, finira par s'attaquer aux institutions de l'Etat si ce dernier n'arrive pas à l'éradiquer. La menace n'est donc pas à sous-estimer.

Dans un Etat qui se veut émergent dans la décennie à venir, la sécurité des personnes et de leurs biens devait non seulement être une priorité des autorités publiques, mais surtout être une réalité pour chaque individu. Ceci démontre qu'aucun phénomène perturbateur de la paix sociale dans le pays, ne devrait être négligé. Plusieurs sont ceux qui s'interrogent pour comprendre la montée en puissance des kulunas, au point de tenir la police nationale et l'ensemble des services de sécurité dont dispose le pays, en échec. Chaque congolais en général et Kinois en particulier voudrait savoir ce qui peut expliquer l'impuissance de l'action répressive du gouvernement dans la lutte anti-kuluna depuis la politique de tolérance zéro décrété par le ministère de la justice depuis $2009^{4}$. N'est-il pas opportun d'envisager d'autres approches éradicatrices au regard des limites constatées? Une interrogation que la présente communication voudrait dans une approche multidisciplinaire et de façon transversale, répondre en proposant des pistes de solution pour l'éradication effective et durable du phénomène kuluna qui constitue un véritable défit sécuritaire pour la RDC.

\section{Bref aperçu sur la genèse du phénomène}

Généralement les études situent la naissance du phénomène Kuluna dans les années 2006-2007, dans la fièvre de premières élections générales de la troisième République, avec l'affrontement des partisans des candidats aux élections présidentiels ${ }^{5}$. Si le phénomène en soi est apparu pendant cette période électorale, ses racines se trouvent dans la crise des années 90 et le pillage qui s'en était suivis mettant l'économie du pays par terre et créant des multiples chômeurs ${ }^{6}$. La criminalité urbaine en RDC est une conséquence de la chute de l'économie nationale à la fin du régime autoritaire du président fondateur. Les professionnels du crime se présentaient dès lors comme des victimes d'une mauvaise gestion de la situation socio-politique qui s'était répercuté sur l'économie. Le gouvernement de l'époque

2 MUSSU FARAJA, L'ONU et les groupes armées dans la sous-région de grands lacs, mémoire de Licence, Université officielle de Bukavu, 2009, p.37.

3 Phénomène Kuluna « Banditisme urbain », sur www.diademesdafrique.net.

4 Tolérance zéro : Luzolo Bambi s'attaque aux kuluna, sur www.gabonesco.com, consulté le 21 septe :bre 2018

5 MALUNGILA Christian, La répressions actes commis par la personne dit Kuluna au regard de la procédure pénale congolaise, Mémoire de Licence, Université de Kinshasa, 2012, p.28.

6 Processus démocratique en RD Congo 1990-2010, sur www.cheikfitanews.net; En RDC la crise est aussi économique, sur https://mobile.lemonde.fr, consulté le 22 septembre 2018. 
préoccupé par sa survie à tout prix, négligea d'apporter des réponses adéquates à cette problématique. C'est la mauvaise gestion de la délinquance urbaine et notamment celle des jeunes qui est à la base de ce qu'on a qualifié en 2006 et 2007 de phénomène kuluna.

\section{Descritpion des kulunas}

Les kulunas sont des jeunes dont l'âge varie entre 18 à 25 ans, pratiquant des arts martiaux (judo et karaté), ayant des touillages sur le corps et passant la grande partie des journées dans consommation des chanvres mêlés des boissons alcoolisés notamment « agene » et 《zododo $»^{7}$. Contrairement aux « chegués » traditionnels, les kulunas disposent des domiciles et sont des responsables des familles. Ils n'attaquent pas souvent les habitants de leurs quartiers qui sont probablement leurs parents, frères ou sœurs. En contrepartie, ces derniers n'osent pas les dénoncer, craignant d'une part les représailles, et d'autre part par le risque d'attaque de leur quartiers par les kulunas d'autres communes en cas d'arrestation de ceux vivant avec eux, car les kulunas se livrent par fois aux véritables guerres de territoires entre gangs. Ils opèrent avec les armes blanche, notamment les couteaux, les lames de rasoirs, les tournevis, les bouteilles, mais surtout avec l'arme caractéristiques des kulunas qui est la machette qu'ils aiguisent pour terroriser la population. Les actes posés vont du vol qualifié à l'assassinat des personnes qui résistent à exécuter leur ordre de donner l'argent et les biens réclamés.

\section{B. Causes du phénomène kuluna}

Parmi les causes à l'origine du phénomène l'on peut citer notamment, le chômage, les drogues, l'abandon familiale à la suite d'une accusation de sorcellerie, l'instrumentalisation politique des jeunes et la pauvreté des parents.

\section{Le manque d'occupation rémunératrice}

Le constat démontre qu'au Congo-Kinshasa, les riches deviennent plus riches et les pauvres devienne plus pauvres, rendant inévitable la révolte des pauvres victimes de cette injustice économique. Une cause principale de la vulnérabilité de la jeunesse congolaise, le chômage est le facteur majeur du phénomène kuluna ${ }^{8}$. Plusieurs kulunas affirment s'être lancé dans la criminalité pour des raisons économiques. Ils ont été contraints de chercher à gagner leur vie en semant la souffrance dans la population, sans quoi ils ne peuvent survivre. Certains d'entre eux sont des véritables responsables ayant des femmes et enfants à supporter à tout prix. En dehors de ces voies de fait, les kulunas n'ont pas une autre possibilité de gagner leur vie.

7 Kinshasa : le phénomène kuluna constitue un danger, sur www.afrique.congotimes.info, consulté le 13 septembre 2018.

8 Les origines du phénomène kuluna, sur www.congoforum.be, consulté le 13 septembre 2018. 
De ce point de vue, les kulunas apparaissent comme des victimes d'un apartheid économique qui caractérise la République Démocratique du Congo (RDC) ${ }^{9}$. La répartition non équitable de la richesse nationale, conduit à la frustration de la majorité de la population qui se sent en marge du pays sans espoir. Dans l'histoire des peuples, la confiscation de la richesse nationale, a toujours conduit à la révolte des victimes de cette injustice sociale ${ }^{10}$. C'est dire ainsi que, le vrai problème est la bonne gouvernance qui doit éradiquer la ségrégation économique dont les jeunes sont majoritairement victime.

Dans ce sens l'on peut affirmer que l'Etat lui-même crée un environnement favorable à l'insécurité grâce à son mauvais système économie. La ville de Kinshasa est l'une des villes africaines où les immeubles poussent comme des champignons et dont les dernières marques des véhicules de luxe augmentent chaque jour davantage, alors que le budget de l'Etat est le plus faible d'Afrique. Cela veut dire que la richesse de l'Etat se partage entre un groupe des individus bénéficiaires des privilèges du pouvoir public et ayant adoptée la malversation financière comme mode de gouvernement, se comportant en véritables affairistes. Le phénomène kuluna constitue une réaction des citoyens lésés par la société injuste. C'est ainsi que même dans leur modes opératoire, les kulunas ne s'attaquent pas à une personne l'apparence misérable. Ils doivent remarquer les signes de richesse (bijoux, gonflement des poches, change en publique devises étrangères, etc.) sur un individu avant de l'attaquer $^{11}$.

\section{La consommation des drogues.}

A côté des kulunas à motivation économique, il s'observe une autre catégorie des kulunas par fait d'entrainement. C'est le cas de ceux qui, sans nécessairement être contraint de se radicaliser dans la criminalité pour survivre, ont intégré le groupe par la consommation des drogues avec les kulunas. A force de fréquenter les criminels, on finit soi-même à devenir criminel. Cette carterie se constitue des jeunes disposants des parents capables de les prendre en charge, mais lèvent l'option de se mettre hors la loi. Ils sont même recherchés par leurs parents qui, parfois les font arrêtés pour une rééducation carcérale en vue de leur réinsertion sociale. Il s'agit en quelque sorte des enfants prodigues ayant quitté les toits familiaux sans raisons valables. Pour cette gâterie, la criminalité est considérée comme une distraction, un passe-temps et non un métier.

9 Isaac MAYELE, les principales cause et perspectives de développement pour la lutte contre la pauvreté urbaine à Kinshasa, Kinshasa 2008, p.74.

10 Département des affaires étrangères de la Confédération Suisse, rapport économique annuel sur la RDC, Kinshasa 2017, p. 10.

11 Le phénomène Kuluna ou la violence des jeunes, CODESRIA, juin 2015. 


\section{La pauvreté des parents}

La pauvreté des parents est l'une des principales causes de l'abandon familial, conduisant les responsables des familles (pères) à déserter leurs obligations familiales d'assurer l'encadrement et l'éducation de leurs enfants. Encore une fois, c'est la mauvaise gouvernance économique qui est responsable de la pauvreté parentale galopante des parents congolais. Depuis l'époque de la Zaïrianisation ${ }^{12}$ qui donna lieu à expropriation des entreprises aux expérimentés gestionnaires blancs, pour les donner aux novices gestionnaires Zaïrois ; plusieurs entreprises tombèrent en faillites mettent plusieurs parents dans le chômage. Le pillage des années 90 acheva les reste de ce qu'on pouvait qualifier de l'économie nationale, faisant des plusieurs parent des désœuvrés, sans emplois, susceptible de leur permettre d'assurer la prise en charge de leurs enfants. C'est dire que les kulunas sont des victimes de l'Etat, lui-même victime d'une gestion prédatrice.

Il importe de souligner par ailleurs la contribution des églises dites de réveille dans la déstabilisation des familles à Kinshasa. Faisant une véritable concurrence avec les débits des boissons dans l'augmentation en nombre, les églises de réveille sont à la bases de dislocation de plusieurs familles, à travers les accusations gratuite de sorcellerie à l'égard des enfants $^{13}$, amenant les parents à se débarrasser de ces derniers. Parmi elles, l'apport du ministre de combat spirituel de « Maman Olangi » peut être souligné avec son fameux examen de l'état d'âme. Cette pratique a conduit des centaines des milieux des enfants dans la rue et qui ont finis par se transformer en kulunas dans la quête de leur survie ${ }^{14}$. L'on devrait donc lutter contre cette pratique dans les églises, car la loi sur protection de l'enfant garantie la protection des droits de l'enfant.

\section{Les défis explicatifs de l'échec de l'action policière anti-kuluna}

Dans l'exécution de sa mission traditionnelle d'assurer la protection des personnes et de leurs biens, la police nationale congolaise (PNC) est confrontée à plusieurs difficultés ou défis qui contribuent à son inefficacité sur terrains. S'agissant de la lutte anti-kuluna, les défis de la PNC sont d'une part endogènes et exogène d'autres parts. Parmi les défis endogènes, l'on peut signaleur, le manque des moyens logistiques, techniques et stratégiques, ainsi que la complicité des certains policiers agissant de concert avec les kulunas, considéré comme leurs mèches. Les défis exogènes, sont notamment la corruption de certains magistrats instructeurs et la récupération politique du phénomène.

12 De la décolonisation à la Zaïrianisation, sur www.cairn.info, consulté le 11 septembre 2018.

13 OFPRA, les enfants accusés de sorcellerie à Kinshasa, novembre 2015, p.3.

14 Note 13. 


\section{Le manque des moyens logistiques et stratégiques.}

La PNC est l'une des polices archaïques et la moins équipée de l'Afrique des grands Lacs. Elle manque les moyens matériels et financiers nécessaires pour la permettre d'accomplir sa mission. Les pickups devant assurer la mobilité policière, sont à ces jours à compter au bout de doits et en mauvaise état ${ }^{15}$. En outre les zones opératoires des kulunas et leurs lieux de refuges sont parfois inaccessibles par véhicules et exigent les motos pour y accéder, que la police ne dispose presque pas, en dehors de quelques motards qui accompagnent les cortèges des autorités politiques. Les matériels d'équipement personnel des policiers ne sont pas régulièrement mis à la disposition de ces derniers pour permettre une action efficace. La logistique de la police vas jusqu'au salaire qui est parmi les plus dérisoire des fonctionnaires de l'Etat. C'est dire que le policier étant misérable, ne peut être efficace dans l'exécution de sa mission avec une motivation nécessaire pour traquer les semeurs de troupes dont les kulunas et assurer la securité de la population avec plus d'abnégation. Sans une logistique adéquate et une motivation suffisante. Une chose est de confier la mission de sécurisation des personnes et leurs biens, une autre est d'y mettre les moyens nécessaires.

Il est également important de rappeler le déficit stratégique de la politique dans la lutte contre le phénomène kuluna. Elle manque le professionnalisme suite a l'insuffisant de formation dans le chef de plusieurs policiers. La plupart d'entre eux n'ont pas finis les études primaires et ne sont probablement pas bien formés à cette profession. Par ailleurs, il n'est pas exclu que l'retrouve parmi les policiers, les anciens kulunas, ne disposant aucune déontologie policière. La remise à niveaux des policiers est donc un préalable à la modernisation d'une police capable de faire face aux éventualités de la société qu'est appelé à protéger.

\section{La complicité des policiers avec les kulunas}

Il s'observe paradoxalement que certains policiers sont des mèches de kulunas, en agissant de concert avec eux et en assurant leur protection lors des opérations ${ }^{16}$. Lors des opérations nocturnes officiellement qualifiées de patrouilles, les policier qui croisent les kulunas revenants de leurs forfaits ou qui les surprennent en action ne les arrêtent pas, mais partagent avec eux les butins. De ce point de vue la police est en quelque sorte contributrice à la monté en puissance du phénomène. Conscient de cette complicité, les kulunas n'ont aucune crainte de la police dont ils savent qu'ils sont des oiseaux de même plumage, mais à qui la faute ? Sans partir par quatre chemins, l'Etat est à la base de ce paradoxe, du fait de n'avoir pas assuré un traitement digne pouvant assurer la dignité des policiers dans l'exécution sa mission honorable.

15 Raoul KIENGE-KIENGE et Sara LIWERANT (dir), Violence urbaine et réaction policière à Kinshasa (Rd. Congo). Sens et non-sens, Academia l'Harmattan, Louvain-la-neuve, 2017, p145.

16 Les Kuluna, gangs de jeunes qui sévissent à Kinshasa - RDC - RFI [archive], 28 novembre 2013. 
III. La corruption de certains magistrats instructeurs des dossiers mettant en cause les kulunas

La police et la population se dénonce souvent les cas des kulunas transmis aux parquets pour être instruits et fixés devant le juge, mais ne connaissent pas une suite satisfaisante du fait que dans moins de 48 heures, les délinquants arrêtés circulent dans les mêmes quartiers où ils avaient commis des forfaits. Le clientélisme et la corruption des certains magistrats instructeurs, alourdissent le travail de la police qui apparait comme faisant du surplace dans un cercle vicieux et donc sa capacité opérationnelle s'affaiblis davantage faible face à la monté en puissance du phénomène kuluna. De même, ceux qui exceptionnellement peuvent être condamnés et emprisonnés, ne s'inquiètent pas, car les conditions des centre pénitentiaires congolais n'ont rien d'intimidant ou de rééduquant et les kuluna y sortent plus délinquants qu'avant l'incarcération.

\section{La récupération politique du phénomène kuluna}

Considéré au départ comme un phénomène social, les kulunas ont finis par devenir des véritables acteurs politiques, recrutées dans les ligues de jeunes des partis politiques, ils sont politiquement utile à plusieurs titres : ils assurent la securité de certains hauts placés de leurs partis, et lors des compétions électorales, ils sont envoyé pour troubler les manifestations des adversaires politiques ${ }^{17}$. Dans la province du Congo central, le gouverneur les avait utilisés pour attaquer l'assemblée nationale et empêcher les députés provinciaux de voter une motion contre lui et son gouvernement. A Kinshasa, ils sont également utilisé pour perturber les revendications des syndicats et autres fonctionnaires qui dérangent les leaders politiques membres du gouvernement contre qui les syndicalistes voudraient s'adressé. A ce titre le phénomène bénéficie d'une forte protection politique et son démentiellement ne sera jamais effective sans une véritable volonté politique de ceux qui l'entretient après l'avoir récupérer pour l'utiliser dans le combat politique. Cette protection se manifeste souvent lors de leurs arrestations, les pressions politiques sont parfois exercées sur les Officiers de Police Judiciaires (OPJ) et magistrats instructeurs de leurs dossiers, pour leur libération ${ }^{18}$.

\section{Tentatives d'une nouvelle approche gouvernementale éradicatrice}

La lutte anti-kuluna n'aboutira pas si les véritables causes à son origine n'ont pas été résolues. Un combat n'est jamais gagner lorsqu'on ne connait pas son véritable adversaire. Au regard du diagnostic qui viens d'être préalablement fait, le gouvernement est appelé à adopter une nouvelle approche d'éradication du phénomène kuluna. Pour mettre définitivement

17 Héritier BAHATI MUJANGI, Le phénomène « Kuluna » ou la violence des jeunes, un défis pour la gouvernance sécuritaire de la ville de Kinshasa, CODESRIA, 2011, p.8.

18 Note 15, p.143. 
fin au phénomène national kuluna, il importe d'envisager la récupération de ces jeunes professionnel du crime par la formation ou le recyclage des kuluna, l'amélioration des conditions sociales des parents notamment par les allocations familiales, l'adoption d'une politique d'encadrement de la jeunesse désœuvrée.

\section{La formation professionnelle ou recyclage des kulunas}

Point n'est besoins de rappeler que la majorité des kulunas, le sont pour des raisons économiques faute d'une occupation rémunératrice qui les permettent de de gagner leur vie par le travail. La formation étant un préalable à l'accès à l'emploi, la réinsertion sociale de ces jeunes délinquants passe par un apprentissage professionnel initié à leur intention en vue de leur recyclage. Mais avant cette formation, un dialogue doit être créé entre le gouvernement et les kulunas pour définir de manière concertée le processus de démobilisation dans le banditisme.

Il revient à l'Etat de prendre en charge cette formation professionnelle et créer des débouchés d'emplois pour leur absorption. Pour ce faire la promotion des industries et des petites et moyennes entreprises devraient également être pris en compte dans cette absorption des anciens kulunas qui sortiront des instituts de préparations professionnels. La répression ayant montrée ses limites, l'Etat ne pourra mettre fin à ce phénomène qu'en orientant vers des horizons qui pourrons assurer leur survie de façon digne et honorable. Un jeune qui est occupé à exercer son travail dont il est sûr d'avoir une rémunération capable de lui assurer sa prise en charge, n'aura jamais le temps de basculer dans la criminalité.

\section{La réduction de la pauvreté des parents}

L'Etat doit lutter contre la pauvreté des parents afin de permettre à ces derniers d'assurer eux-mêmes l'éducation et la formation de leurs enfants. Parmi les kulunas, il y en a qui ont quitté leurs familles à cause de la misère qui règne dans leurs maisons. Un enfant qui passe deux jours sans mangé, n'aura pas d'autre choix le troisième jour que d'aller se débrouiller seul pour trouver un marceau de pain, à croquer. La pauvreté des parents a donc un impact significatif sur l'avenir des enfants et contribue à la recrudescence de la criminalité ou de la délinquance juvénile. Le gouvernement devrait ainsi lutter contre ce fléau notamment par l'allocation familiale aux familles qui présente le niveau le plus élevé de la pauvreté, afin d'assurer la protection et l'encadrement des enfants.

\section{L'adoption d'un plan national d'encadrement de la jeunesse}

La solution durable au phénomène kuluna passe également par un plan national d'encadrement des jeunes, à court moyens et long terme avec l'implication des tous les acteurs concernés dans la protection de la jeunesse. La faiblesse des stratégies initiées jusqu'ici pour lutter contre le phénomène kuluna est la concentration de tous les efforts du gouverne- 
ment sur la solution répressive, qui à causes des contraintes relevées a montré ses limites. La conjugaison des efforts tripartites entre le gouvernement, la police et les familles est indispensable pour une solution effective de ce fléau. Chaque composante devrait jouer réellement son rôle dans la riposte contre cette criminalité juvénile.

\section{La famille}

Elle doit rester le milieu le plus propice pour l'épanouissement des enfants et leur première éducation. Il faut souligner à ce stade que la stabilisation des foyers passe par une planification familiale. Car, l'étude révèle que la surpopulation favorise la criminalité, comme c'est le cas à Kinshasa. En effet, c'est dans les quartiers les plus peuplés de Kinshasa que l'on remarque le taux le plus élevés de la criminalité, comme dans les communes formant le district de la Tshiangu que l'on appelle aussi « chine populaire ». D'où la nécessité d'une sensibilisation des familles à la planification familiale, pour une meilleure prise en charge des enfants.

\section{La police}

Outre le renforcement de capacité, la moralisation du personnel policier et son équipement; la collaboration avec la population dans la lutte contre l'insécurité urbaine reste l'ultime objectif pour l'efficacité de l'action policière. Cette collaboration passe par un dialogue entre les policiers et les citoyens. L'objectif de ce dialogue est le rétablissement de la confiance des citoyens dans la police qui est pour l'instant inexistant du fait des divers failles de la police dans ses différentes opérations et qui a laisser croire à une grande majorité de la population que les kulunas agissent de connivence avec la police. Il s'agit d'un défis supplémentaire de la police, car même si elle peut être bien équipée et dotée des moyens conséquents, sans une certaine complicité entre la population et la police il sera difficile d'éradiquer le phénomène kuluna. Cette foi de la population dans la police ne devrait pas être un cadeau, mais plutôt le fruit d'un effort supplémentaire dans le respect strict de la loi qui impose aux policiers un professionnalisme remarquable.

\section{Le gouvernement}

Comme l'on peut le constater la grande responsabilité dans cette lutte contre le phénomène kuluna revient au gouvernement de la république. A ce stade il sied de souligner d'entrer de jeu que la gratuité de l'enseignement primaire prônée par la constitution ne doit pas rester une lettre morte ${ }^{19}$. Le gouvernement est appelé à y accorder une particulière attention en assurant son effectivité afin de permettre à chaque enfant de bénéficier de mêmes droits dès le bas âge pour baliser le chemin à son épanouissement. Une politique de prise en charge 
des enfants issues des familles vulnérables devraient être mise en place pour alléger la charge des foyers les plus pauvres.

Le contrôle du taux développement humain doit être contrôlé par une politique préventive de lutte contre la surpopulation. Ainsi, un recensement général s'impose pour une bonne répartition de la richesse nationale par le nombre d'habitant. Il importe par ailleurs de rappeler que la ville de Kinshasa connait une surpopulation dû à l'exode rurale, qui appel en effet à une modernisation des villes et villages congolaises, permettant l'épanouissement de chaque congolais en général et les jeunes en particuliers à partir de son milieu natal. Il s'agit donc d'établir un contrôle de circulation et de déplacement interne des personnes. Par ailleurs le plan urbanistique de la ville de Kinshasa devrait être reformé et améliorer par la création des nouveaux quartiers dans les espaces vides notamment dans les communes de N'sele et maluku pour permettre le désengorgement du centre-ville.

\section{CONCLUSION}

Le phénomène kuluna incarne de nos jour la violence juvénile dans les grandes villes congolaises et particulièrement à Kinshasa et la police nationale assiste impuissante à la montée en puissance de la terreur de ce fléau en dépits des multiples opérations mort-nées contre du dit phénomène. Etant fruit d'un mauvais système économique de l'Etat congolais lui-même victime d'une gestion prédatrice, le phénomène kuluna n'est plus à éradiquer par la répression qui a montré ses limites d'une part, et confrontée à plusieurs défis de l'autre. Une suppression efficace et durables cette maladie sociale, passe par la résolution des causes à l'origine de son émergence. Il revient au gouvernement de tirer les conséquences de l'échec de la politique répressive et envisager une politique non répressive de récupération des kulunas en vue de leur réinsertion sociale à travers un encadrement susceptible de leur permettre de gagner la vie autrement que la violence.

\section{BIBLIOGRAPHIE}

1. De la décolonisation à la Zaïrianisation, sur www.cairn.info.

2. Département des affaires étrangères de la Confédération Suisse, rapport économique annuel sur la RDC, Kinshasa 2017, p. 10.

3. Héritier BAHATI MUJANGI, Le phénomène «Kuluna» ou la violence des jeunes, un défis pour la gouvernance sécuritaire de la ville de Kinshasa, CODESRIA, 2011, p.8.

4. Isaac MAYELE, les principales cause et perspectives de développement pour la lutte contre la pauvreté urbaine à Kinshasa, Kinshasa 2008, p.74.

5. JORDC, ${ }^{\circ} 5$ février 2011, constitution de la République Démocratique du Congo

6. Kinshasa : le phénomène kuluna constitue un danger, sur www.afrique.congotimes.inf o.

7. Le phénomène Kuluna ou la violence des jeunes, CODESRIA, juin 2015. 
8. Les Kuluna, gangs de jeunes qui sévissent à Kinshasa - RDC - RFI [archive], 28 novembre 2013.

9. Les origines du phénomène kuluna, sur www.congoforum.be.

10. MALUNGILA Christian, La répressions actes commis par la personne dit Kuluna au regard de la procédure pénale congolaise, Mémoire de Licence, Université de Kinshasa, 2012, p.28.

11. MUSSU FARAJA, L'ONU et les groupes armées dans la sous-région de grands lacs, mémoire de Licence, Université officielle de Bukavu, 2009, p.37.

12. OFPRA, les enfants accusés de sorcellerie à Kinshasa, novembre 2015, p.3.

13. Phénomène Kuluna « Banditisme urbain », sur www.diademesdafrique.net,

14. Processus démocratique en RD Congo 1990-2010, sur www.cheikfitanews.net; En RDC la crise est aussi économique, sur https://mobile.lemonde.fr.

15. Raoul KIENGE-KIENGE et Sara LIWERANT (dir), Violence urbaine et réaction policière à Kinshasa (Rd. Congo). Sens et non-sens, Academia l'Harmattan, Louvain-laNeuve, 2017, p145.

16. Rapport circonstanciel sur l'insécurité en RDC, AZADHO, Kinshasa 2011, p.5.

17. Tolérance zéro : Luzolo Bambi s'attaque aux kuluna, sur www.gabonesco.com. 